onset, the other 78 patients $(75.7 \%)$ developed glomerulonephritis after a median of $3(1 ; 12)$ months. Hematuria was present in $94(91.3 \%)$, proteinuria in 85 patients $(82.5 \%)$, nephrotic syndrome in 11 patients $(10.7 \%)$, hypertension in 31 patients (30.1\%). Rapidly progressive glomerulonephritis (RPGN) defined by the doubling of serum creatinine within $\leq 3$ months developed in 29 patients $(28.2 \%$ of 103$)$. Myeloperoxidase-ANCA-positive patients $(n=12)$ developed RPGN significantly more often than proteinase-3-ANCA-positive patients $(n=78): 7(58.3 \%)$ vs 22 $(28.2 \%)(p=0.0499) .11(10.7 \%)$ patients developed AKI, stage 3 being the most common (in 8 patients). 40 (38.8\%) patients exhibited indolent renal disease course, i.e. without worsening of renal function. By the end of the follow-up, 33 $(32.0 \%)$ patients were diagnosed with CKD grade $3 b-5$, among them 11 patients (10.7\%) developed end-stage renal disease (ESRD). 6 patients (5.8\%) died. As the first line induction therapy all patients received corticosteroids in combination with cyclophosphamide in 93 patients $(90.3 \%)$, rituximab (RTX) in 3 patients $(2.9 \%)$, mycophenolate mofetil in 7 patients $(6.8 \%)$. RTX was used for remission induction in $27(26.2 \%)$ patients due to refractory or recurrent disease. During follow-up, 74 patients $(71.8 \%)$ developed one or more severe relapses with a relapse rate of 0.27 per patient-year. $50(48.5 \%)$ patients had renal relapses (0.096 per patient-year).

Conclusions: Prevalence of renal involvement in the studied group was lower than expected, most likely because of a large proportion of patients with localized GPA. Our study showed that RPGN was not the most common feature of renal involvement. Almost $40 \%$ of patients developed indolent course of kidney disease. Nevertheless one third of patients developed CKD G3B or worse by the end of the follow up. Despite effective induction therapy with high-dose corticosteroids, cytotoxic agents and/or RTX relapses, both renal and extrarenal, are frequent.

Disclosure of Interest: None declared

DOI: 10.1136/annrheumdis-2017-eular.5104

\section{FRI0347 RHEUMATOID FACTOR IS CORRELATED WITH DISEASE ACTIVITY AND INFLAMMATOTY MARKERS IN ANTINEUTROPHIL CYTOPLASMIC ANTIBOTY-ASSOCIATED VASCULITIS}

S. Watanabe, T. Gono, K. Nishina, N. Sugitani, E. Watanabe, H. Yabe, C. Terai. Rheumatology, Jichi Medical University, Saitama Medical Center, Saitama, Japan

Background: Raised levels of serum rheumatoid factors (RFs) of different immunoglobulin classes had been reported in a high proportion of patients with rheumatoid vasculitis. RF used to be studied and was shown elevated in some forms of vasculitides. Patients with antineutrophil cytoplasmic antibody (ANCA)-associated vasculitis (AAV) are often positive for RF. However, the clinical significance of RF has been seldom examined in AAV.

Objectives: The aim of this study was to investigate association between the presence of RF and clinical features and outcomes in AAV.

Methods: Eighty-one patients were diagnosed with AAV from 2006 to 2015 in our hospital. Among 81 patients, forty-seven patients $(17$ males, median age 67 years) who were not complicated with rheumatoid arthritis and in whom RF was measured before the treatment, were studied, retrospectively. Patients were classified using the European Medicines Agency vasculitis classification algorithm. AAV included eosinophilic granulomatosis with polyangitis $(n=10)$, granulomatosis with polyangitis $(n=14)$, microscopic polyangitis $(n=16)$ and unclassifiable vasculitis (UV) ( $n=7)$. Patients with UV with MPO-ANCA or PR3-ANCA were included in this study. IgM-RF was measured using a latex agglutination assay. Disease activity was assessed with Birmingham vasculitis activity score (BVAS). IgM-RF, C-reactive protein (CRP), erythrocyte sedimentation rate (ESR), serum ferritin, IgG, IgM, IgA, MPO-ANCA and PR3-ANCA were obtained from hospital records. Clinical manifestations between RF-positive subset $(n=29)$ and RF-negative subset $(n=18)$ were analyzed using Fisher's exact and Wilcoxon rank sum tests. Correlation coefficients were established with Spearman's correlation coefficient. Data were shown as medians (interquartile range).

Results: BVAS was higher (14 (12-22) vs $12(6-16), P=0.026)$ in the RF-positive subset than the RF-negative subset. CRP and ESR were higher $(P=0.020$ and 0.007 , respectively) in the RF-positive patients. IgM-RF titers significantly correlated with BVAS $(r=0.50, P=0.0004)$. In addition, CRP, ESR, IgM and IgG also had a significant correlation with IgM-RF titers. The frequency of initiation of dialysis therapy (14\% vs $6 \%)$, usage of mechanical ventilation (14\% vs $0 \%)$ and mortality ( $10 \%$ vs $0 \%$ ) were higher in the RF-positive subset than in the RF-negative subset although no significant differences were shown.

Conclusions: In AAV, IgM-RF titers are significantly correlated with disease activity and inflammatory markers. Presence of RF could be a poor prognostic factor in patients with AAV.

References:

[1] Watts R, Lane S, Hanslik T, et al. Development and validation of a consensus methodology for the classification of the ANCA-associated vasculitides and polyarteritis nodosa for epidemiological studies. Ann Rheum Dis 2007;66:222227.

[2] Westedt ML, Herbrink P, Molenaar JL, et al., Rheumatoid factors in rheumatoid arthritis and vasculitis. Rheumatol Int 1985;5:209-14.

[3] Kronbichler A, Kerschbaum J, Gründlinger G, et al. Evaluation and validation of biomarkers in granulomatosis with polyangiitis and microscopic polyangiitis. Nephrol Dial Transplant 2016;31(6):930-6.

Disclosure of Interest: None declared
DOI: 10.1136/annrheumdis-2017-eular.4555

\section{FRI0348 ASSOCIATED INFLAMMATORY SYNDROMES IN TAKAYASU'S ARTERITIS: MANY FACES OF A DISEASE}

S.N. Esatoglu ${ }^{1}$, A.M. Celik ${ }^{2}$, D. Ucar ${ }^{3}$, A.F. Celik ${ }^{4}$, S. Ugurlu ${ }^{1}$, G. Hatemi ${ }^{1}$, M. Melikoglu ${ }^{1}$, I. Fresko ${ }^{1}$, V. Hamuryudan ${ }^{1}$, H. Ozdogan ${ }^{1}$, S. Yurdakul ${ }^{1}$, H. Yazici ${ }^{1}$, E. Seyahi ${ }^{1}$. ${ }^{1}$ Istanbul University, Cerrahpasa Medical School, Department of Internal Medicine, Division of Rheumatology; ${ }^{2}$ Istanbul University Cerrahpasa Medical School, Department of Internal Medicine; ${ }^{3}$ Istanbul University, Cerrahpasa Medical School, Department of Ophthalmology; ${ }^{4}$ Istanbul University, Cerrahpasa Medical School, Department of Internal Medicine, Division of Gastroenterology, Istanbul, Turkey

Background: Case reports and series suggest that Takayasu's arteritis (TA) can co-exist with various inflammatory disorders.

Objectives: We conducted a formal study to look specifically at the frequency of inflammatory disorders and symptoms in a large cohort of TA followed by a single tertiary center.

Methods: There were 238 patients registered with a diagnosis of TA. Of these, 19 died, 18 were lost to follow-up and 3 did not wish to response our questionnaire. The remaining 198 patients were called back at the outpatient clinic. A standardized form sought whether the patient was also diagnosed as inflammatory bowel disease (IBD), ankylosing spondylitis (AS), Behçet's syndrome (BS), amyloidosis, uveitis, rheumatoid arthritis (RA), systemic lupus erythematosus (SLE), systemic sclerosis (pSS), Sjögren syndrome, psoriatic arthritis, inflammatory myositis, small vessel vasculitis, autoimmune/demyelinating or any other inflammatory disorder.

Results: Overall, 198 (175 F/ 23 M) patients were studied. The mean age at the time of TA diagnosis was $34 \pm 12$ years. Subclavian artery was the most common involved artery (84\%), followed by common carotids $(78 \%)$ and aorta (65\%). Currently, while $25(16 \%)$ patients were off treatment, $72(47 \%)$ patients were using glucocorticoids, 47 (31\%) azathioprine, $32(21 \%)$ methotrexate and $44(29 \%)$ biological agents.

We identified in total $37(19 \%)$ patients with inflammatory diseases (IBD: $n=12$; AS: $n=15$; and BS: $n=10$ ). Table shows their demographic characteristics. Among the remaining 161 patients, the most frequent feature was inflammatory back pain $(36 \%)$, followed by recurrent oral ulcers $(15 \%)$, erythema nodosum $(11 \%)$, arthritis $(10 \%)$, papulopustular lesions $(7 \%)$, uveitis $(4 \%)$, and genital ulcer $(1 \%)$. It was noted that inflammatory back was mostly located on the dorsal area. Regarding autoimmune diseases, we also observed RA $(n=3)$, psoriasis $(n=2)$, autoimmune hepatitis $(n=2)$, SS $(n=1)$ and SSc $(n=1)$.

Table 1. Demographic features of 37 TA patients with inflammatory bowel disease (IBD), ankylosing spondylitis (AS) or Behçet's syndrome (BS)

\begin{tabular}{lcccc}
\hline Concomitant disease & F/M & $\begin{array}{c}\text { Mean age at TA } \\
\text { diagnosis (SD) }\end{array}$ & $\begin{array}{c}\text { Mean age at concomitant } \\
\text { disease (SD) }\end{array}$ & $\begin{array}{c}\text { Time of TA diagnosis } \\
\text { in relation to } \\
\text { concomitant disease }\end{array}$ \\
\hline AS $(n=15)$ & $13 / 2$ & $31 \pm 8$ & $27 \pm 11$ & $\begin{array}{l}\text { Simultaneous }(n=3) \\
\text { TA preceded }(n=7) \\
\text { AS preceded }(n=5) \\
\text { Simultaneous }(n=9) \\
\text { TA preceded }(n=1) \\
\text { IBD preceded }(n=2) \\
\text { IBD }(n=12)\end{array}$ \\
& $11 / 1$ & $33 \pm 9$ & $31 \pm 9$ & $\begin{array}{l}\text { Simultaneous }(n=5) \\
\text { TA preceded }(n=1) \\
\text { BS preceded }(n=4)\end{array}$ \\
BS $(n=10)$ & $7 / 3$ & $35 \pm 13$ & $32 \pm 13$ &
\end{tabular}

Conclusions: TA does co-occur with IBD, AS or BS in about $1 / 5$ of the patients, at least in a hospital setting and without a clear temporal pattern. This could be due to the close association of TA with MHC class-1 diseases. In addition, the high prevalence of inflammatory back pain in the dorsal spine in TA needs further scrutiny.

Disclosure of Interest: None declared

DOI: 10.1136/annrheumdis-2017-eular.6276

\section{FRI0349 THE SNP RS6871626 LOCATED IN IL12B REGION MAY INFLUENCE ON VASCULAR LESIONS OF TAKAYASU ARTERITIS}

T. Nakajima ${ }^{1}, \mathrm{H}$. Yoshifuji ${ }^{1}, \mathrm{C}$. Terao $^{2}, \mathrm{~K}$ Murakami $^{1}, \mathrm{R} . \mathrm{Nakashima}^{1}$, Y. Imura ${ }^{1}, \mathrm{~K}$. Ohmura ${ }^{1}$, T. Mimori ${ }^{1}$. ${ }^{1}$ Department of Rheumatology and Clinical Immunology, Graduate School of Medicine, Kyoto University; ${ }^{2}$ Center for Genomic Medicine, Kyoto University, Kyoto, Japan

Background: Takayasu arteritis (TAK) is a type of large vessel vasculitis, which affects aorta and its main branches. We previously found a single nucleotide polymorphism (SNP), rs6871626 located in IL12B region, as a susceptible gene to TAK and reported that the risk allele at the SNP is associated with the risk of aortic regurgitation $(A R)^{1)}$. However, there have been no studies on the association of the SNP with the organ involvements other than AR in TAK patients.

Objectives: To investigate the association of the SNP with arterial and organ involvements except for AR.

Methods: We examined the medical records of 85 patients with TAK, stratified 